\title{
Implementation of Control System and Tracking Objects in a Quadcopter System
}

\author{
Siva Ariram ${ }^{1(\bowtie)}$, Juha Röning ${ }^{1}$, and Zdzisław Kowalczuk ${ }^{2}$ \\ ${ }^{1}$ Biomimetics and Intelligent Systems Group (BISG), \\ University of Oulu, Oulu, Finland \\ siva.ariram@oulu.fi \\ ${ }^{2}$ Gdansk University of Technology, Gdansk, Poland
}

\begin{abstract}
In this paper, we implement a quadcopter assembly with control and navigation module. The project also includes the design of the control panel for the operator which consists of a set of the microcontroller and the glove equipped with sensors and buttons. The panel has a touch screen which displays current parameters such as vehicle status, including information about orientation and geographical coordinates. The concept of quadcopter control is based on the movement of the operator hand. In addition, we have included the object detection for detecting the objects from the quadcopter view of point. To detect an object, we need to have some idea of where the object may be and how the image is divided into segments. It creates a kind of chicken and egg problem, where we must recognize the shape (and class) of the object knowing its location and recognize the location of the object knowing its shape. Some visual characteristics such as clothing and the human face, they can be part of the same subject, but it is difficult to recognize this without recognizing the object first.
\end{abstract}

Keywords: Drone $\cdot$ Quadcopter $\cdot$ Kalman filter $\cdot$ GPS $\cdot$ IMU

\section{Introduction}

The widespread use of drones in the present times and the desire to use the current knowledge acquired by students during their studies inspired the team of authors to create their own flying vehicle and supply it with an appropriate control program. Starting from defining the concept of drone systems and qualifying them in accordance with the applied construction, the principles of quadcopter operation and maneuvering are presented. Next, we describe examples of realizations available on the market, including their goals, as well as the scope of this work.

One of the most important issues in automation (control) is to estimate the pose (posture) of the quadcopter, which includes the inclination, tilt and rotation angles, called roll, pitch and yaw. The estimation of the pitch and roll angles is obtained by means of the operation of two independent Kalman filters, and the yaw angle is obtained thanks to the gyroscope data integration operation. On the basis of the current measurements and set value, an error is calculated, which is given to the input of the proportional-integral-derivative (PID) regulator [1]. The controller generates the control signal in the PWM form, which is then sent to the motorized actuators. 
Computer simulation simulating the operation of designed complementary filters using gyroscope and accelerometer readings was carried out. Due to the fact that the results obtained from the supplementary filters were not satisfactory, we decided to implement the Kalman filter, which improved the efficiency of the system and gave us satisfactory results. That is why we have implemented this KF solution in the reported project. Finally, several tests were carried out to stabilize and control the system of the flying vehicle, allowing controlled rotation in 3 axis.

\subsection{System Description}

The operation of the developed system is based on communication between quadcopter control modules. The operator panel is a module with diagnostic and control functions. The module consists of an electronic system equipped with a touch screen displaying parameters such as the current quadcopter states (modes) and geographical coordinates. This operator's kit will also be equipped with a button responsible for activating the engines, changing the speed of the engines and activating certain control modes. The device will be powered by a lithium-polymer battery. The principle of system operation is presented in Fig. 1.

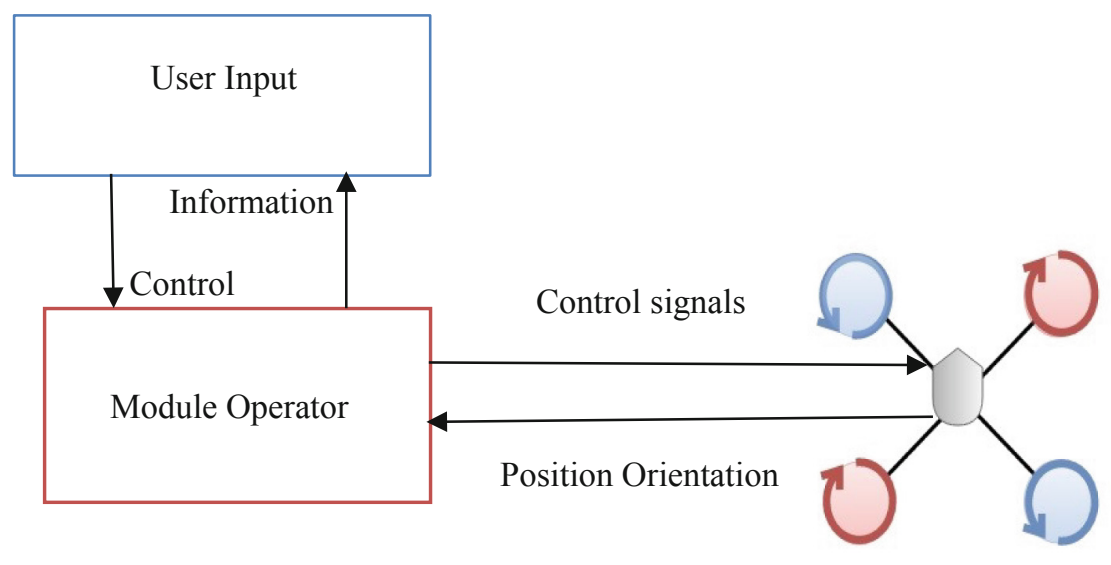

Fig. 1. Basic Quadcopter architecture

A part of the quadcopter and the operator's panel has been found on the market. The project uses: lithium-polymer batteries, RC apparatus, 4x motors, 4x ESC regulators, propellers, voltage converters, IMU sensors, microcontrollers, radio systems and a GPS pick box. Using the above-mentioned parts, a new quadcopter and the operator panel were assembled.

The assembly process of the vehicle has been divided into two parts. In the first part, a subset of the frame was considered. The ESC regulators were then attached to the arms with clamps and the motors were fastened with screws. In the lower part of the central lobe using a Velcro, a battery was attached, while the upper part contained a microcontroller, a GPS receiver, a radio system, an RC apparatus receiver and an IMU system. 


\section{System Implementation}

One of the main problems in the implementation of the project is to determine the current orientation of the copter. The estimation of the vehicle pose consists in determining the values of the angle of inclination, deflection and rotation (roll, pitch, and yaw). For this purpose, a gyroscope and accelerometer were used. To eliminate the disadvantages associated with such sensors, special filters have been implemented that combine (fuse) measurements.

This section shows the results of estimating only the value of the deviation angle, while the designed system uses orientation estimates in all axis.

\subsection{Gyroscope}

A sensor was used to read the data, which was placed on a quadcopter that was initially immobilized and stood on a flat surface. A graph of the angular velocity measurement measured with a Y-axis gyroscope is shown in Fig. 2.

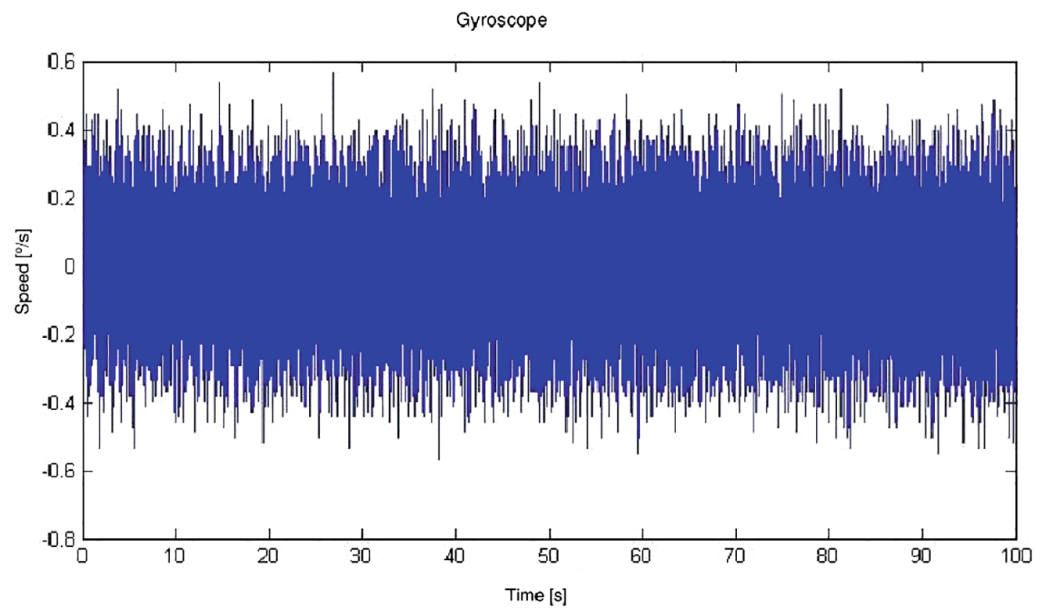

Fig. 2. A graph showing the angular velocity in the $\mathrm{Y}$ axis read using the gyroscope.

In Fig. 2, it is confirmed that the rate measured with a gyroscope is noisy, with noise reaching values from $-0.5 \%$ to $0.5 \%$. Having gyroscopic angle data, it is possible to obtain information on Roll, Pitch and Yaw angles $(\varphi, \theta, \psi)$ through integration. The total angular velocity shown in Fig. 2 leads to the Pitch value $(\theta)$. The integration result is shown in Fig. 3.

The results obtained after integration are clearly characterized by lower noise levels. It can be seen from Fig. 3 that despite the fact that the vehicle was stationary 


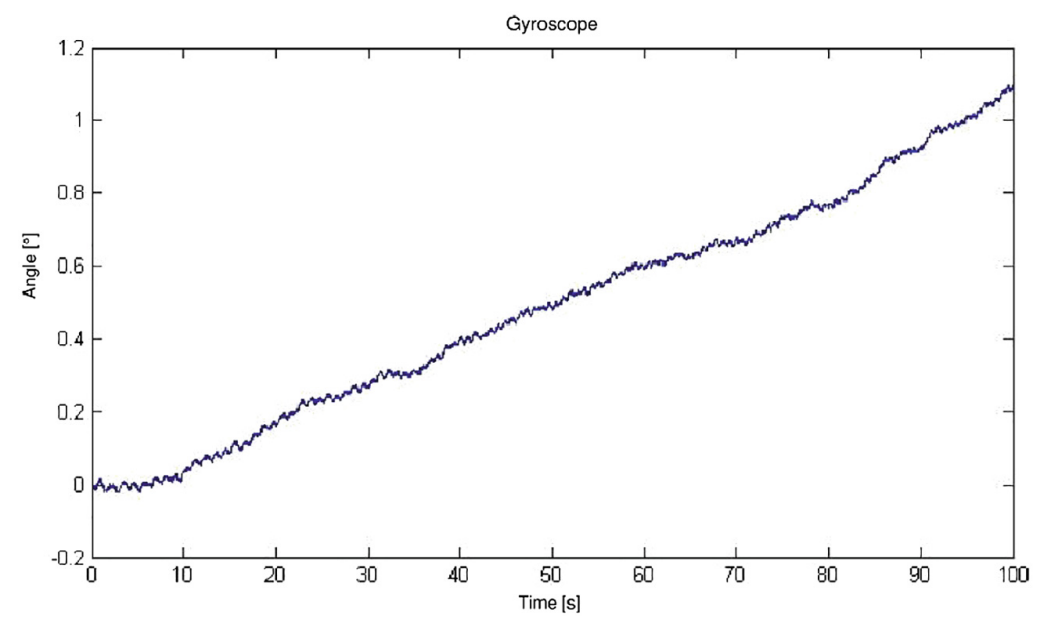

Fig. 3. A graph showing the angle $\theta$ after the angular rate integration.

during the measurements, the estimated value changed by about $1.1^{\circ}$ in $100 \mathrm{~s}$. This phenomenon is called drifting. This is the main defect of the gyroscopes, forcing compensation by means of data from other sensors.

\subsection{Accelerometer}

The acceleration sensor returns the acceleration values along the 3 axis $\left(a_{x}, a_{y}, a_{z}\right)$. Based on this data and the appropriate trigonometric operations [2], the roll and pitch angles defining the rotation of the quadcopter along the $\mathrm{X}$ and $\mathrm{Y}$ axis can be determined. The obtained acceleration rates $\left(a_{y}, a_{z}\right)$ are shown in Fig. 4.
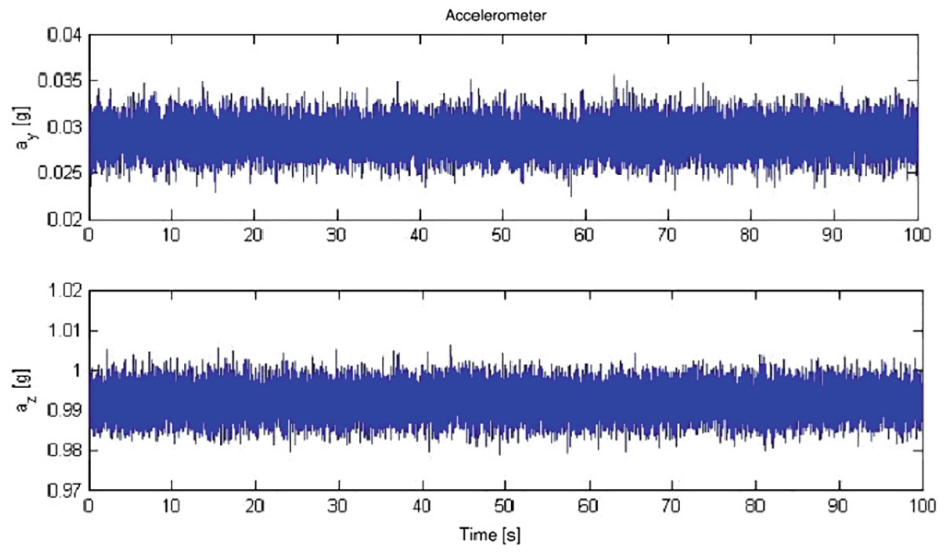

Fig. 4. A graph showing the values of accelerations $\left(a_{y}, a_{z}\right)$ read from the accelerometer. 
Also to determine the pitch value $(\theta)$, acceleration measurements from the 3 -axis accelerometer were used. Considering quadcopter measurements (as in the case of flat accelerations), because the accelerations had no other components besides gravitational acceleration, the total sum of accelerations $\left(a_{x}, a_{y}, a_{z}\right)$ is $1 \mathrm{~g}$. The angle of the pitch was determined based on the data shown in Fig. 3, and the results are shown in Fig. 5.

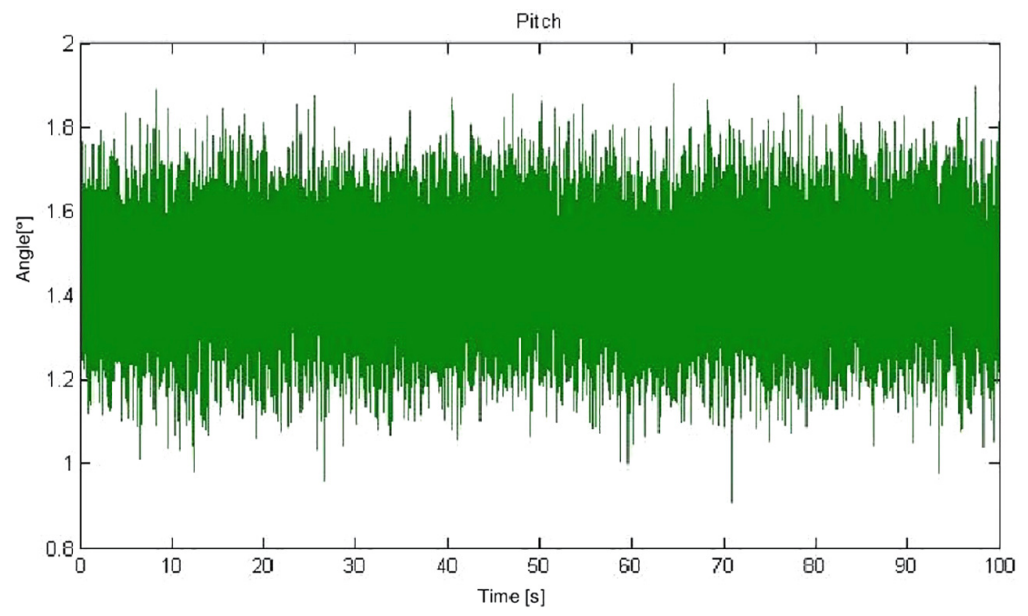

Fig. 5. The value of $\theta$ is calculated by means of trigonometric transitions.

By performing the appropriate trigonometric transformations, the Pitch value was obtained. Let us consider that the calculated angle is not affected by drift, which occurs in the case of gyroscopic measurements, but is generally characterized by higher noise. The obtained non-zero angle results from the non-ideal alignment of the sensor during measurements.

\subsection{Kalman Filter}

The Kalman filter is a linear estimate of the state model [2] and is modeled by means of state:

$$
\begin{gathered}
X_{k+1}=A x_{k}+B u_{k}+W_{k} \\
Y_{k}=H x_{k}+Z_{k}
\end{gathered}
$$

Where $\mathrm{A}, \mathrm{B}, \mathrm{H}$ denote the state, input and output matrices respectively, $\mathrm{X}$ is the state vector, $\mathbf{u}$ is the input, $\mathrm{Y}$ is the measured value, $\mathrm{W}$ is the process noise and $\mathrm{Z}$ is the measurement noise. The Kalman filter assumes that the measurement and process noise are not correlated with each other and their average values are zero.

The Kalman filter procedure is divided into two stages. The first stage is called the time update. The second stage of the Kalman algorithm is the update of measurements [2]. Figure 6 represents a graph showing the operation of the Kalman filter for a stationary angle. 


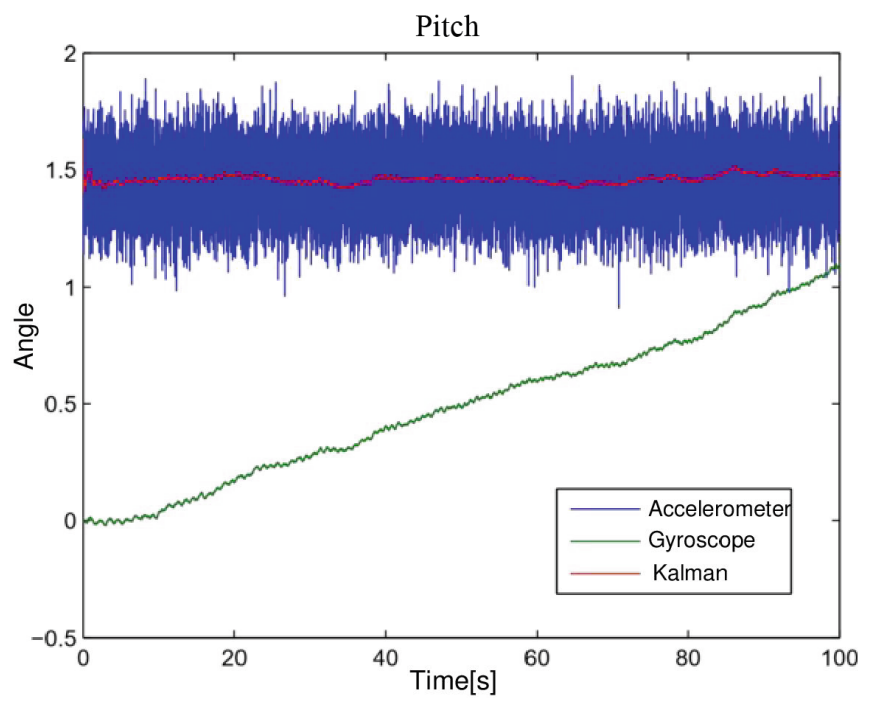

Fig. 6. The result of the operation of the Kalman filter for a stationary case.

The Kalman filter eliminates the initial gyro error and suppresses the noise from the accelerometer. The operation of the Kalman filter for a stationary case gives similar results to the complementary filter. The Kalman filter result for the rapidly changing angle is shown in Fig. 7.

Pitch

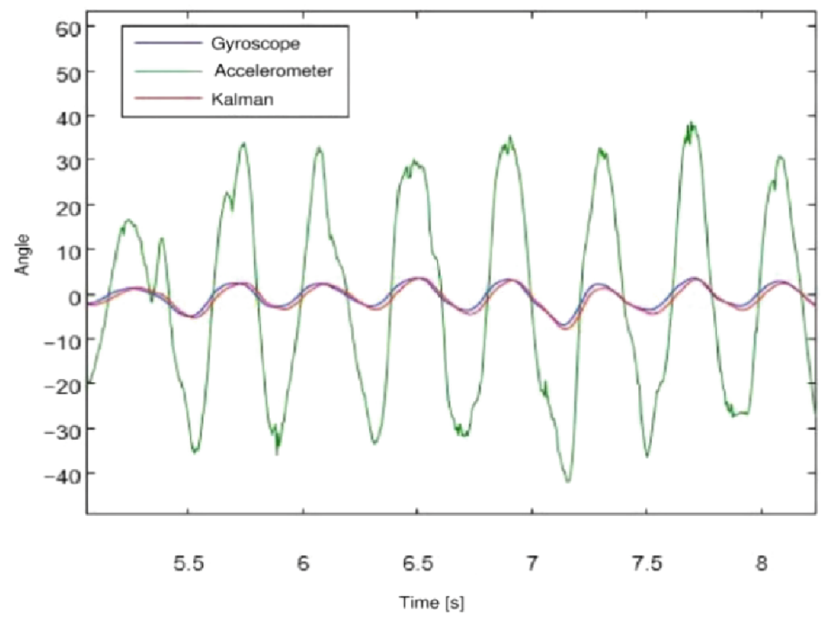

Fig. 7. The result of the Kalman filter operation for rapid angle changes. 
Based on Fig. 7, it can be seen that the results obtained using the Kalman filter have a much lower delay (in phase) and show less impact of the accelerometer noise than the one obtained using the accelerator. Finally, a measurement test was carried out to compare the performance of the complementary filter and the Kalman filter when the quadcopter is rotated at different angles. A fragment of the obtained series of measurements is shown in Fig. 8.

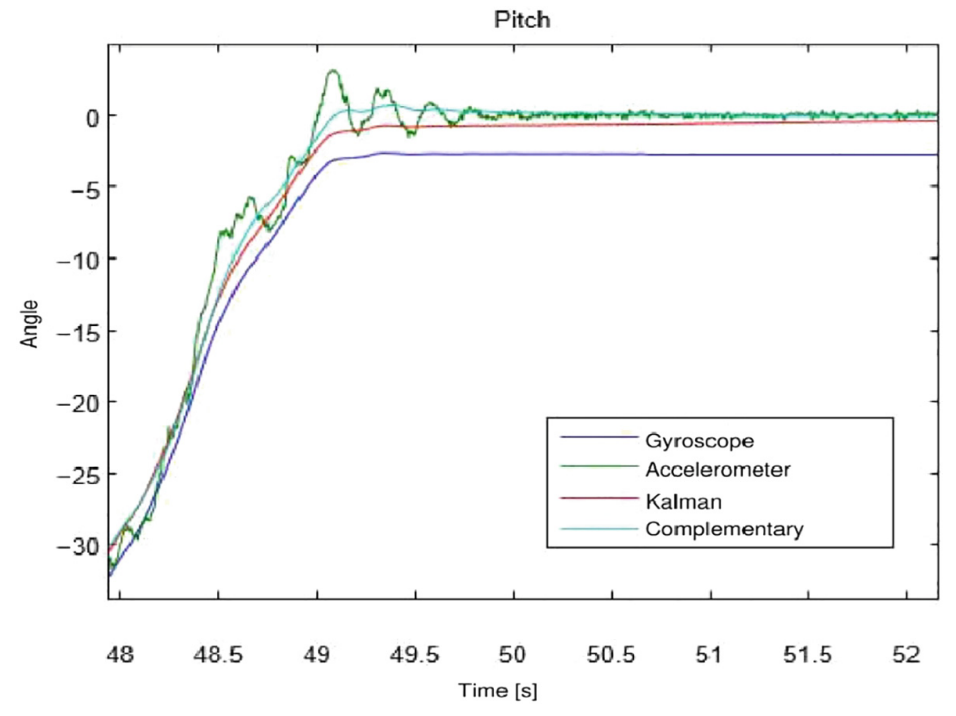

Fig. 8. A graph showing the operation of the Kalman filter.

In conclusion, both filters are suitable for use in systems where the angle changes at low frequencies, while in systems with rapid changes it is recommended to use the quadcopter system based on the Kalman filter. In this project, two separate Kalman filters were used to determine the pitch and roll angles, and gyroscope measurements were used to estimate the yaw angle. Orientation estimation has been implemented both in the quadcopter and in the operator's module.

\subsection{Control Module}

The quadcopter control module consists of a microcontroller to which external circuits are connected. The diagram of these connections is shown in Fig. 9.

The microcontroller memory has implemented control algorithms and functions that communicate with various systems, such as an IMU sensor, a GPS receiver, a radio system, an RC receiver, a PC computer and engine controllers. By transforming the information coming from the IMU sensor, the microcontroller determines the current orientation of the quadcopter. On the basis of the vehicle configuration and the parameter set coming from the operator module, a corresponding control command is calculated, which is given to the engine controllers in the form of PWM signals (with appropriate 
filling) [3]. The control signals are only calculated if the user triggers a corresponding signal to activate the motors by means of a brake. Based on the data from the GPS receiver system, the control module determines the GPS coordinates. Communication with a PC allows you to read the data needed to simulate the operation of algorithms on real sensor readings [8].

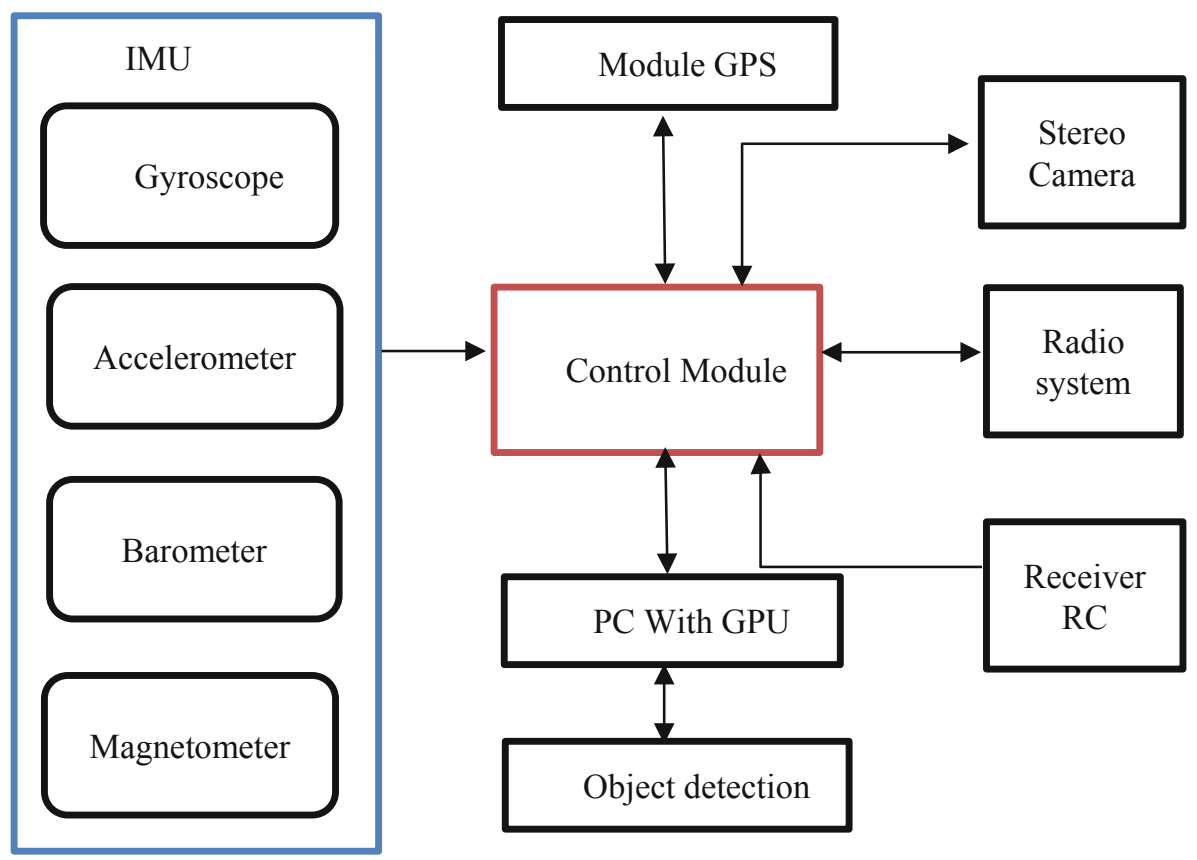

Fig. 9. Diagram of connections of the control module with external circuits.

\subsection{UAV Communication}

The Ground station and UAV had bi-directional communication between them. The ground station plans the aerial path of the UAV vehicle and keeps the UAV updated. The communication was carried out with the help of a UART serial port. The implemented communication allows for transferring data from the quadcopter sensors to the computer system, concerning cameras, angular speeds, linear accelerations, and coordinate positions and others calculated for systemic purposes [10].

\section{Experimental Results}

\subsection{Stability Testing}

At the very beginning it was checked how the quadcopter stabilizes in one axis. For this purpose, the set point value of the considered angle was fixed to 0 . The vehicle was 
prepared for testing by pulling a string through the center of the frame and attaching one end to its railing, while the other end was held by the operator. Initial tests were performed using a standard RC apparatus. The completed aerial vehicle is shown in Fig. 10.

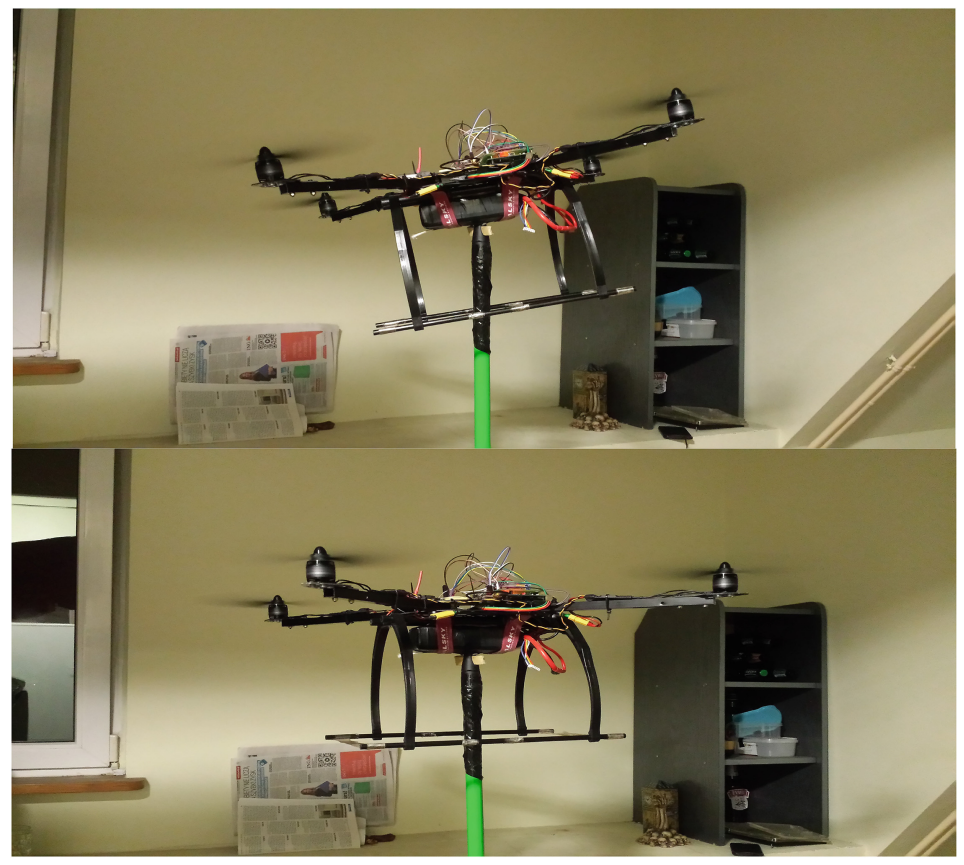

Fig. 10. Testing Stabilization with quad-copter.

The purpose of the test was to check the correctness of the implementation of control algorithms and orientation estimation. After selecting the appropriate PID controller settings, stabilization of the quadcopter was observed. In this way, the test was completed successfully. The next test was an attempt to stabilize the quadcopter in two axis by selecting the appropriate parameters of the PID controllers. In this case, it was possible to move the platform into a specific position. Initial values of integrating and switching elements were set to 0 [4].

The experimental results confirmed the known PID theory. Initially, only the proportional gain was tuned. In the cases when the gain was too small, the quadcopter slowly returned to the steady state, but it was unable to reach it (which caused the regulation to fail). Gradually, with the increase in the factor $\mathrm{P}$, it was noticed that the position of the quadcopter began to stabilize. In the case of excessive gain, oscillations around the steady state were were generated [5, 7]. After determining the proportional gain value, the values of differential and integrating factors were tuned. The differentiating part affected the speed of the quadcopter reaction, while the integrating element reduced the error of the steady state. 


\subsection{Controllability Testing}

The final phase of the tests consisted of controlling the quadcopter with the help of a glove. For this purpose, the vehicle is placed on the platform. During the tests, the operator checked if the quadcopter reacts to the control signals coming from the squeeze. At the beginning, the operation of 4 buttons was checked. According to the assumptions, the vehicle respectively: activated the engines, changed the speed of rotation of the engines and activated the manual control mode. Next, the operator, holding the pressed control button, turned the palm. Quadcopter at that time replicated the operator's movements.

\subsection{Object Detection}

When it comes to precision, the results are promising. We have shown how to train the system, and that images obtained through the data acquisition system can be used to detect objects as part of motion detection, also showing the effectiveness of the adopted methods [6]. In a few cases, the system detected more objects than those associated with annotators embedded in the original data. However, they have been marked as false positives (false alarms), with a clear classification to the appropriate class of objects using visual inspection. The object detection from the view of quadcopter is shown in Fig. 11.

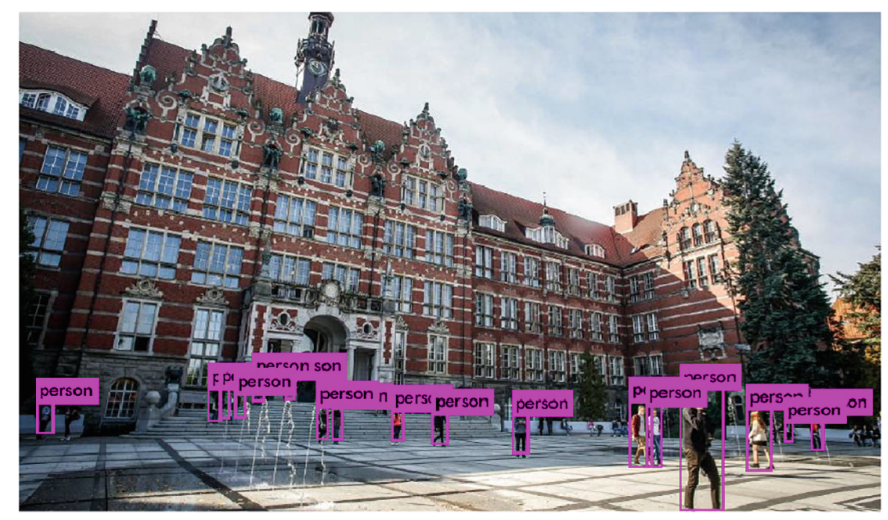

Fig. 11. Detection from the view of quadcopter.

The proposed detection system was taught according to VOC and COCO data sets for five classes of possible objects on the road (pedestrians, cars, buses, bicycles and motorcycles) instead of using 20 classes characteristic of the VOC data set. In detecting/assessing the vehicle, our detector only generates car detection data (evidence) without taking into account the bus detection data, because buses are assigned to the MISC or trucks in the KITTI data set. When vehicles are detected within five classes, objects with a similar appearance, such as an airplane or a boat, are treated as a background. This increases the effectiveness of the SoftMax classification, which may slightly improve the performance of this system. Pedestrians had also slightly improved 
detection efficiency, with the exclusion of animal classes (such as cat, cow and horse). We also evaluated the effectiveness of the detection methods, including the background class. Our experimental results have shown that the six-class method, with the background class, surpasses the five-class method [9].

\section{Conclusion}

In summary, the project concerns the construction of a quadcopter system and equipping it with a navigation module and a control module. The designed quadcopter is able to perform basic maneuvers such as elevation, descent, forward tilt, lateral deviation and rotation around the central vertical axis.

An important issue considered in this project and significant during the control was the estimation of the quadcopter's state and its pose (orientation), which consisted in determining the slope, deviation and rotation angles (referred to as roll, pitch and yaw). A number of tests were also carried out to verify the accuracy of the implemented system, including its algorithms and methods.

\section{References}

1. Pedley, M.: Tilt sensing using a three-axis accelerometer. Free. Semicond. Appl. Note 1, 2012-2013 (2013)

2. Romaniuk, S., Gosiewski, Z.: Kalman filter realization for orientation and position estimation on dedicated processor. Acta Mechanica et Aautomatica 8(2), 88-94 (2014)

3. McCarron, B.: Low-Cost IMU Implementation via Sensor Fusion Algorithms in the Arduino Environment. California Polytechnic State University, San Luis Obispo (2013)

4. Ylimäki, M., et al.: Fast and accurate multi-view reconstruction by multi-stage prioritised matching. IET Comput. Vision 9(4), 576-587 (2015)

5. Shah, M.K.N., Dutt, M.B.J., Modh, H.: Quadrotor-an unmanned aerial vehicle. Int. J. Eng. Dev. Res. 2(1), 1299-1303 (2014)

6. Kang, M.S., Lim, Y.C.: High performance and fast object detection in road environments. In: 2017 Seventh International Conference on Image Processing Theory, Tools and Applications (IPTA), pp. 1-6. IEEE (2017)

7. Hentati, A.I., Krichen, L., Fourati, M., Fourati, L.C.: Simulation tools, environments and frameworks for UAV systems performance analysis. In: 2018 14th International Wireless Communications \& Mobile Computing Conference (IWCMC), pp. 1495-1500. IEEE (2018)

8. Sagitov, A., Gerasimov, Y.: Towards DJI phantom 4 realistic simulation with gimbal and RC controller in ROS/Gazebo environment. In: 2017 10th International Conference on Developments in eSystems Engineering (DeSE), pp. 262-266. IEEE (2017)

9. Al-Kaff, A., et al.: VBII-UAV: vision-based infrastructure inspection-UAV. In: Rocha, Á., Correia, A.M., Adeli, H., Reis, L.P., Costanzo, S. (eds.) WorldCIST 2017. AISC, vol. 570, pp. 221-231. Springer, Cham (2017).

10. Gasior, P., et al.: Thrust estimation by fuzzy modeling of coaxial propulsion unit for multirotor UAVs. In: 2016 IEEE International Conference on Multisensor Fusion and Integration for Intelligent Systems (MFI), pp. 418-423. IEEE (2016) 